\title{
Construction And Testing of a Bent HeAt PIPE
}

\section{Shuvashish Bhattacharjee ${ }^{1}$, A. N. M. Mizanur Rahman², Saad Bin Abul Kashem}

\author{
${ }^{1}$ Department of Mechanical Engineering, Khulna University of Engineering \& Technology (KUET), Khulna- 9203, BANGLADESH \\ ${ }^{2}$ Professor, Department of Mechanical Engineering, Khulna University of Engineering \& Technology (KUET), Khulna- 9203, BANGLADESH \\ ${ }^{3}$ Lecturer, Faculty of Engineering, Computing and Science, Swinburne University of Technology, Sarawak Campus, MALAYSIA
}

*Email for Correspondence: shuvrome@gmail.com

Cell Phone: (+88) 01714002333

Abstract

Heat pipe was reviewed in former time which was in linear design. But in most cases, the heat pipe is needed in a bent shape. For instance, in a car, the heat transfer way from the engine to the radiator is not in linear. To move heat from parts in a computer i.e. central processing units of the computer may use non-linear heat pipe to reduce the heat generated in it. So the performance analysis for a bent heat pipe is badly needed to fulfill the current demand. In this research, a bent heat pipe was constructed and the performance analysis have been conducted on the bent heat pipe.

Key words

Heat Pipe, Convection Heat Transfer, Evaporator, Condenser

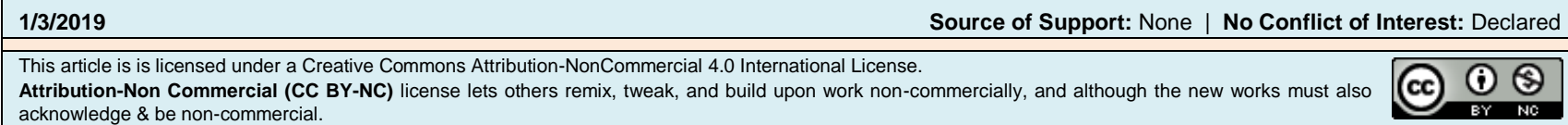

\section{INTRODUCTION}

It is tough and incompetent work in thermodynamics to move heat from one place to another. Sometimesheat carrying losses could occurthat minimizes the total performance? A variety of electronics component's operation depends on the performance of the cooling system because heat is produced there when they operate which need to move away due to perfect as well as good operation. Nowadays the electronics components need to have the ability to be cooled. The cooling process is accomplished by the tiny heat sink attached to it. Cooling problems in microelectric circuits are generally critical because the generated heat must be dissipated from such small surface areas. There are many conventional heat transfer devices. Such as heat exchanger, regenerator, heat pumps etc. Basically, in a heat exchanger, there is one participant who releases heat and there is another participant which gains heat. These two are separated by a solid wall, this two van not be blended at all. They are widely used in many aspects of engineering fields. A car radiator is the familiar model of the heat exchanger which takes heat from the engine and releases it to the air. But fouling factor decreases the efficiency of a heat exchanger. If the fluid flows into the heat exchanger have impurities, it directly hit the wall. The negative side of the heat exchanger is that heat cannot be moved more due to the obligation of heat transfer in that heat exchanger. The negligible thermal conductivity of the fouling film affects the large performance of a heat exchanger. The pumping and maintenance expenses are increased in this way. Another heat transfer device is the regenerator which is more economical in case of components and production. But it has also a disadvantage. The prime drawback of a regenerator always some blending of fluid streams that could not be fully split up. When two fluid streams are allowed to blend, in that case, regenerator can be used. The cooling tower is also a well-known conventional heat transfer device which permits water temperature under the air temperature that is used to cool it. Cautious maintenance is required for it to reduce the risk of water fouling as well as some waterborne organisms. However, in thermodynamics, heat move using heat pipe is most economical and prominent way of heat transfer. The heat pipe has high efficiency and prompt solution of rapid heat transfer in a crucial area. Using a heat pipe the pressure variation is very low. Heat is gained from evaporator section and is released in condenser section. The internal liquid takes heat from the evaporator section and becomes vapor. This vapor moves from heating section to cooling section due to rising of internal pressure and becomes liquid again. Finally liquid returns from cooling section to heating section and thus the cycle is completed. The objective of the present work includes the Construction and the performance test of a bent heat pipe. 


\section{LITERATURE REVIEW}

It is known that heat can be transferred by three modes. These are conduction, convection, and radiation. The conventional heat transfer devices are regenerator, heat exchanger, heat pump etc. However, there are some limitations of these devices. Heat transfer by heat pipe allows the best solution to overcome these limitations. Heat pipe basically instruments where inside fluid takes heat from the evaporator and release heat to the condenser as a result heat transfers. Sometimes heat pipe is said as superconductor due to its ability of maximum \& efficient heat moving rate. Thermo siphon can be an alternative of the heat pipe. But it differs with thermosiphon in that case heat pipe can transfer heat against gravity using evaporation and condensation cyclic process through the wick structure inside it.

The utmost thermal state was found during a scientist named Grover with his assistants were studying on cooling system on nuclear power plant. From then, people use heat pipe to arrange the control of inside temperature.

Heating the water using solar energy could be another example of the heat pipe with the mechanism of evacuated tube solar arrays. In this case, people use distilled water as working fluid which is located inside a copper tube whose end is sealed. Nowadays, flat plate solar water is not used at all because it has up to 40 percent less efficiency than evacuated tube solar heater. In Antarctica, the temperature is extremely cold. There evacuated solar water heater is commonly used to heat the water (Grover, 1964).

There are three parts in a heat pipe names as an evaporator, an adiabatic section, and condenser. Evaporative cooling is offered by it, which helps to transport heat from one location to other location by heating \& cooling of an operating fluid or coolant. Ina heat tube, during heating of the evaporator section by using the heating source, working fluid inside there receives that heat and get hot. This working fluid takes the heat as a result vapor pressure of the liquid is increased. There is a temperature difference between evaporator \& working fluid, so heat from evaporator section is taken by working fluid. The pressure of heater section and the pressure of cooler section are not same at all and due to it vapor moves from heating section to cooling section. Except the heater \& cooler section in a heat tube, the intermediate section is named as an adiabatic section where there is no heat loss or heat gain i.e. heat remains same. In the condenser section, there is also a temperature difference between vapor \& condenser. As a resulting vapor becomes liquid again and because of gravitational force, it again returns to evaporator section using wick structure inside there and the cycle completes. To sum-up, working fluid takes heat from heater section, flow away and finally release heat to cooler section. Then fluid returns again to heater section and the cycle is completed.

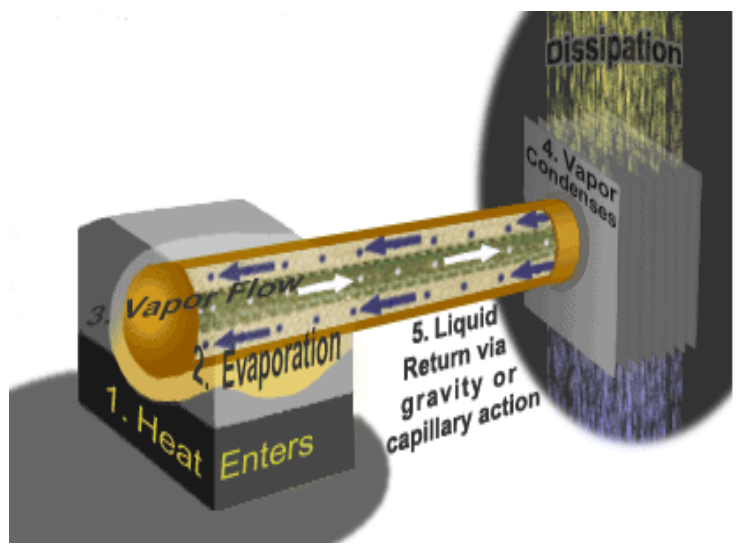

Figure 1: Schematic diagram of a straight heat pipe

Heat uses three modes to transfer. One is conduction, the second is convection and the third is radiation. In conduction heat transfer process energy exchange takes place from the area of higher temperature to that of lower temperature. However, it is restricted to transferring heat only from solid to solid metal. In convention heat transfer process fluid takes heat from a solid body when the temperature of the fluid and that solid body is different, actually, this is the prerequisite that the temperature must be different to transfer heat from one body to another. But it is limited to transfer heat only in case of transferring heat in liquid. Radiation heat transfer process takes place where no medium is needed. However, the radiation energy emitted by the shape or carried in space in the mode of electromagnetic waves according to Maxwell's classic electromagnetic wave theory or in the case of discrete photons according to the Plank's hypothesis (Necati Ozisik).

Gaugleret made a patent on a small weight heat carrying the device with a metallic cover. That is as like as present heat pipe. The metal cover was used as a heat source of the pipe. He used water as a working fluid. Then upon supplying electrical energy the metallic cover became hot \& finally it rejects heat to the pipe by conduction heat transfer process. The pipe then rejected this heat to water by convection heat transfer process. Trefethen in 1962 and 
then Wyatt (1992) with a patent case first suggested a space program related idea in 1963. In the Los Alamos Scientific Laboratory, the concept was suggested by Grover et al. (1970) in late 1963. He also made samples that the momentum was given by this methodology. Grover etal also suggested the name "heat pipe" and summarized that heat pipe could be used as a potential way of heat transfer everywhere. Water was used as operating fluid and heat carrying medium in his liquid sodium heat pipe which was used to be operated up to $110{ }^{\circ} \mathrm{K}$. Another scientists (Haskin and Thomas, 1995) first suggested cryogenic heat pipe in 1966. In October 1964 a person (Hall, 1975) worked in RCA first made a heat pipe which could be temperature controlled. On April 5, 1967, Bienert \& Brennan (1971) at Marcus (1971) at TRW first introduced a differential conducted heat pipe, In Los Alamos Scientific Laboratory a couple of engineers first demonstrated the zero " $\mathrm{g}$ " heat pipe. This helps a lot to resolve the issue of temperature control in a spacecraft. Based on heat pipe theory a lot's of spacecraft has been made either for individual part's temperature control or for the entire structure. Nowadays the requirement of heat pipe has been expanded more and more. Aforementioned heat pipes were in linear axis but as discussed, most heat pipe demands in a bent shape.

\section{Performance Evaluation}

The efficiency of a heat tube depends upon many issues such as maximum heat transfer capacity, operating temperature etc. One of the main performance factors of a heat pipe can be the amount of heat transfer for a specific dimension of a heat tube. If $\mathrm{Q}$ is the heat load on the system then the classic convection equation is-

$Q=A h \Delta t$, Where $\mathrm{h}=$ Convective heat transfer coefficient

$\Delta \mathrm{t}=$ Temperature variation between evaporator and condenser

$\mathrm{A}=$ Area of the container.

\section{Limitations of Heat Pipe}

Despite having so many advantages, there are some drawbacks of the heat tube as well. Sometimes the velocity of vapor from heater portion to cooler portion is not uniform, which is said as a sonic limit. On the other hand, friction occurs because the direction of vapor \& fluid's movement is not same which is said as entrainment limit. The velocity of working fluid from cooler section toward heater section is not as uniform which is said as a capillary limit. Taking the heat from heater section, operating fluid becomes vapor which sometimes cannot be in a uniform way; that is called boiling limit.

\section{Design Considerations}

To choose a specific heat tube for specific heat transfer purpose, some considerations regarding should be taken first. Major parts of a heat tube are Heat tube, operating fluid, and a wick structure. To construct a heat pipe the following steps have to be followed:

- Operating fluid should be chosen which should be compatible with the material of a heat tube i.e. the container.

- Also, acontainer of the heat tube material should be compatible with the operating fluid.

- Operating fluid travel's medium i.e. wick structure should be chosen so that there is no chemical reaction between wick structure \& operating fluid.

Container for example heat tube act as a protector of working fluid from outside which is leak-proofed and. Pressure difference shall be maintained by tube.

Finalization of heat tube may need many issues to be studied which are:

- Suitability (with both the operating fluid and outside environment)

- Ratio of strength with weight

- Ability to transfer the heat promptly

- Should be easy to manufacture

- Permeability

- Wet ability (able to wet as best as possible)

In this work, it was assumed that $1 \mathrm{~m}$ copper pipe would be used as a container. But in the local market $1 \mathrm{~m}$,copper pipe was not available. The found copper pipe had length $0.914 \mathrm{~m}$. That's why a copper pipe of $0.914 \mathrm{~m}$ length was used as a container. Specific working fluid shall be considered for the specific purpose of heat transfer. Because each fluid has it's own temperature ranges. The operation fluid shall be also like that it should be suitable for the heat transfer container. Because if any chemical reaction occurs between container material and operation fluid, there should be a chance of damage of heat transfer. Also, the factor corrosion should be considered. To select an operating fluid one need to consider it's vapor temperature ranges. IN a vapor temperature range, there may be more than one working fluid. To one have to consider which one shall be suitable considering container material, the sole purpose of heat transfer etc. factors. 
The major demands are:

- adjustability with wick and container components

- ability for good heat transfer

- the wet capability of wick and container components

- The vapor pressure should be nominal with working temperature

- Latent heat of evaporation value should be higher.

- The viscous properties of both liquid \& vapor shall be low.

- Surface tension value should be higher.

- Appropriate cooling as well as pouring point

In previous various limitations of heat transfer like viscous, sonic, capillary, entrainment have been discussed which should be considered during finalization of a working fluid. To work against a gravitational force, a heat pipe needs to have high surface tension. In order to get maximum heat transfer, operating fluid need good wet ability with container tube as well as wick structure.

If the operating fluid has a higher value of the latent heat of evaporation, heat can be transferred to the minimum fluid flow. Considering above criteria, availability and no cost of water, it was selected as an operating medium in heat tube. It is needed a capillary operating force when working fluid returns back from cooler section to heater section using wick structure. Heat pipe's ability to transfer the heat mostly depends on the shapes of wick structure. Wick structure should be selected based on the sole purpose of heat transfer and its application area. There are several forms of wick structure by using different material like aluminum, copper, nickel with different pore dimension.

Sometimes some people use ceramics as wick structure in a heat pipe which has a smaller pore magnitude. But ceramics are not flexible to bend which is its drawback. Carbon fiber is also being used broadly as wick material in a heat pipe. Using a carbon fiber as wick structure may result in more heat moving from one place to another. Wick structure shall distribute operating fluid to the all-area of heater section. Operating fluid's selection criteria are mostly the same as wick material choice factors.

High wick thickness \& less pore dimension allow more heat transfer capability. The wick structure should also be suitable with the operating fluid. The operating fluid should be of good wet ability with wick material. Some familiar types of wicks are sintered powder, grooved tube, and screen mesh etc.

In this case, considering above criteria, container material, and working fluid, stainless steel net was used as the wick material.

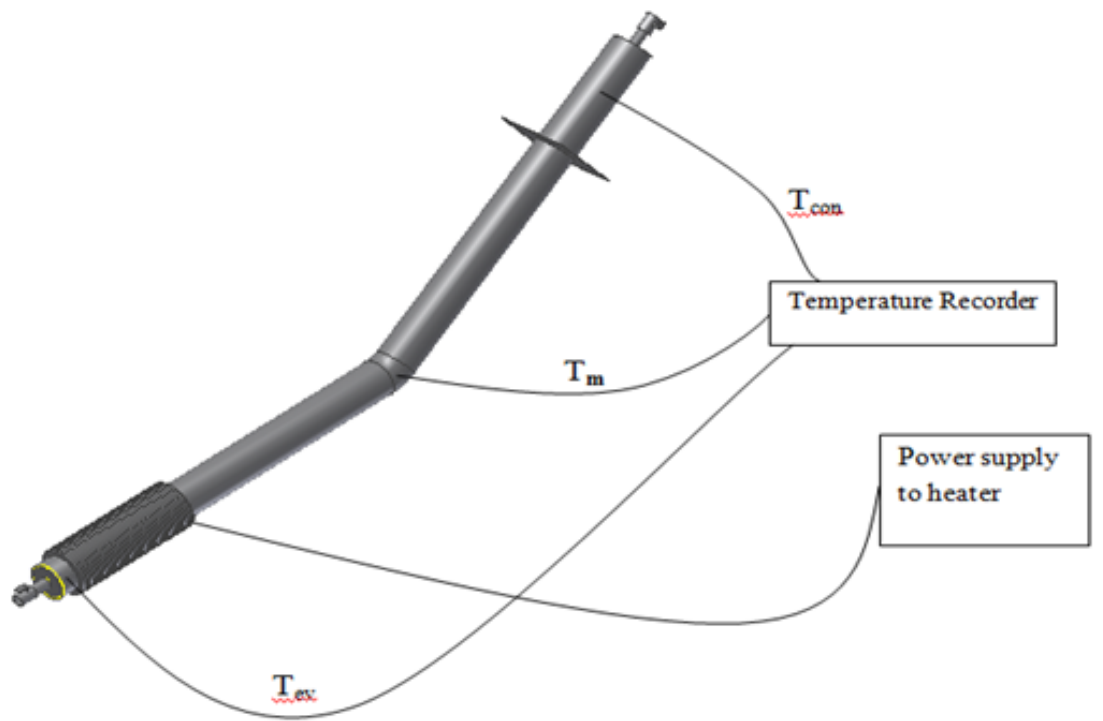

Figure 2: Experimental set up of heat transfer devices

It is needed to use three pieces of $\mathrm{K}$ types thermocouples to calculate the temperature at three points (evaporator, bending point and condenser). These thermocouples are connected to temperature recorder. An aluminum tube is used as heater block. A heating coil of $1500 \mathrm{~W}$ is placed spirally over the aluminum tube. Then the coil was covered by mica and finally by asbestos rope. The coil was connected through a wire to an electrical power source. Two nipples are connected on two holes at the two ends and two gate valves are connected at the end of the nipple. 


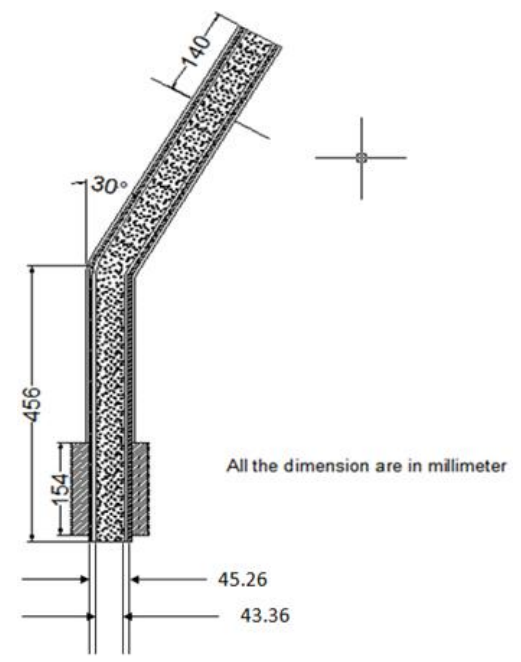

Figure 3: a cross-sectional view of a bent heat pipe.

Following materials are used to construct the bend heat pipe:

- Copper pipe as container-914 mm.

- Water as working fluid

- Stainless steel net as wick material.

- Two 0.5 inch nipples.

- Two 0.5 inch gate valves

- Mika-1 ounce

- Nichrome wire as heater coil-1500W.

- Asbestos rope.

- Glass wool.

- Metal sheet.

\section{Construction Procedure}

The heat pipe is usually made of a tube, sealed at each end, with fluid in it. Because of the availability and no cost of water, it was used as working fluid. A copper pipe (length- $0.914 \mathrm{~m}$ and diameter $0.043 \mathrm{~m}$ ) was used as a container to construct the heat pipe. At first, the copper pipe was bent at $30^{\circ}$. Inside this tube, stainless steel net was used as the wick material. The two ends of the tube were sealed by gas welding using one copper sheet on each end of the tube. One small hole was made on each of the two sheets by drill machine. One nipple ( 0.5 inches) was welled on each of the two holes. Then one get valve was placed on each nipple. A small amount of positive pressure was supplied to the pipe and then each get valve was locked. It was seen that the supplied pressure could not pass from the pipe. This was done to make sure that the copper pipe was completely air tight. After that one gate, thevalve was connected with a vacuum pump for making the copper pipe vacuum. An aluminum tube (length- $0.154 \mathrm{~m}$ and dia$0.045 \mathrm{~m}$ ) was placed on one end of the copper tube which worked as the heater block. This tube was covered by mica to prevent the passing of electricity from the heating coil to the aluminum tube as well as to the copper tube. A heating coil of $1500 \mathrm{~W}$ was spiraled through the aluminum tube. Then the coil was covered by mica and finally by asbestos rope for the aim of insulation. The coil was connected with wire through which electricity can pass through it and finally it was connected with AC source. In the condenser region (length- $0.140 \mathrm{~m}$ and diameter $0.043 \mathrm{~m}$ ) water was supplied through a long pipe to condense the vapor into its liquid region. This long pipe was connected directly to the source. The portion which had been considered as an adiabatic section of the copper tube was covered with glass wool for preventing the transfer of heat. A record of temperatures was kept on the evaporator, bending point and condenser region by using a K-type thermocouple. These thermocouples were connected with temperature recorder. An ammeter (10A-220V) was connected in series connection to measure the current. A variac was used for making a variation in the input power voltage.

\section{EXPERIMENTAL DATA}

Electricity was used to heat the heater block. Water takes away the heat. The rate of flow and temperature of the supplied water were kept constant. The temperature of the supplied water was $28^{\circ} \mathrm{C}$. The rate of flow of the supplied water was .0715 Liter $\backslash$ Sec. Three thermocouples at three different sections were used to measure the temperate at those points; Varick was used to change the voltage of power supply. 


\section{RESULTS AND DISCUSSION}

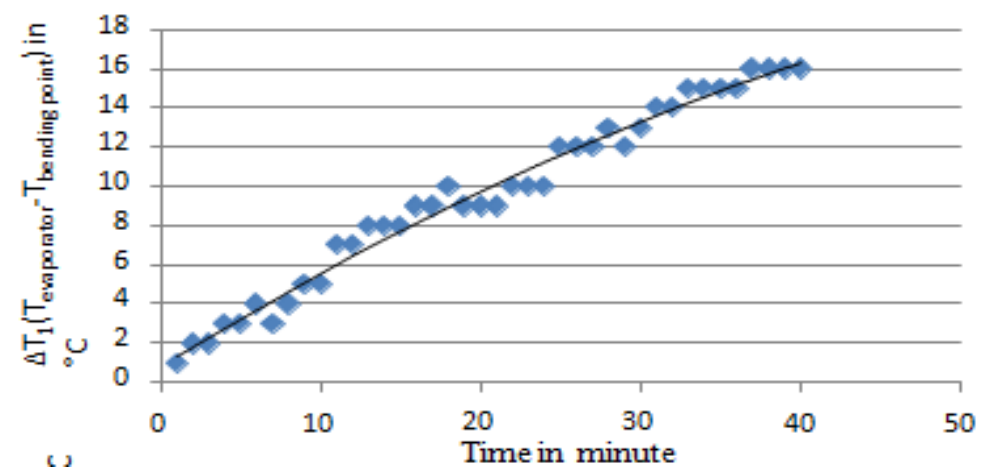

Figure 4: Time vs. $\Delta \mathrm{T}_{1}$

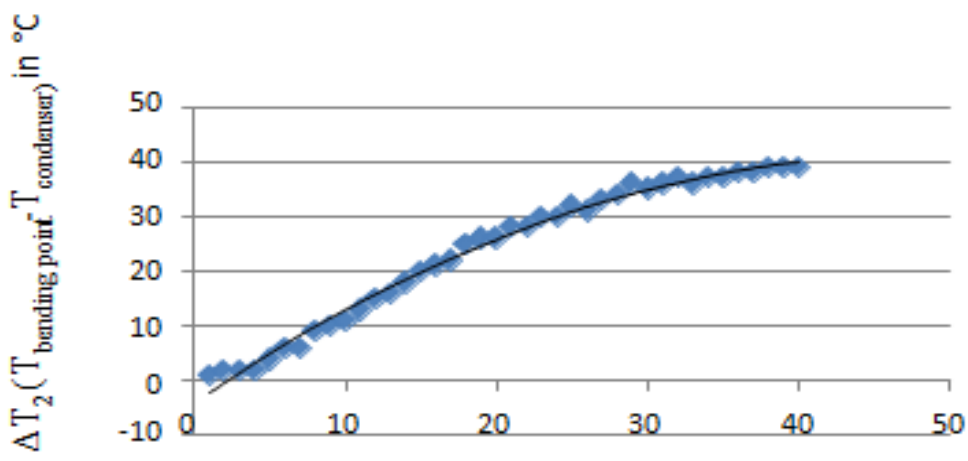

Time in minute

Figure 5: Time vs. $\Delta \mathrm{T}_{2}$

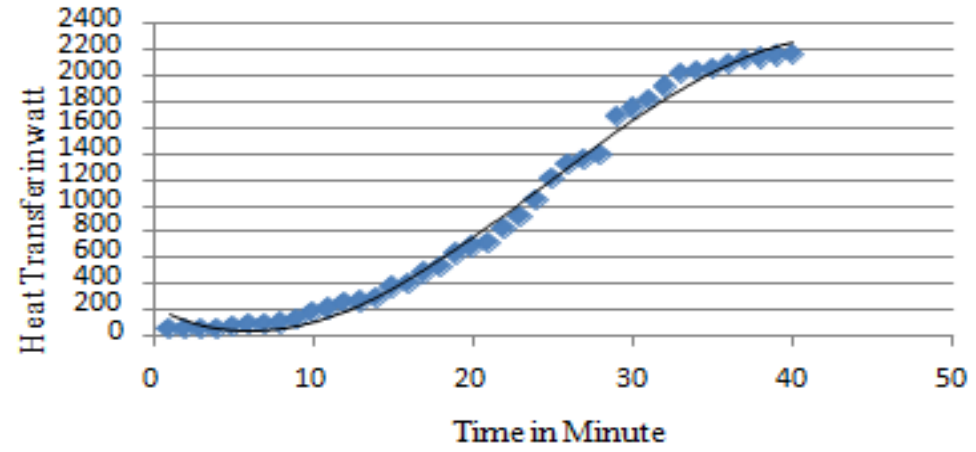

Figure 6: Time vs. heat transfer

The aim of this work was to check the feasibility of the bent heat pipe. It was investigated successfully. Copper pipe was used as a container because of its high thermal conductivity. The working capacity of the heat transfer device is affected by a container used there. The more thermal conductivity of the container makes the best performance.

During sealing the two end of the copper pipe by gas welding, the temperature was very high. As a result, the characteristics of the wick material and the container were not remaining same. During the performance test, the cooling water was supplied by a long pipe which directly connected to the source of water. The cooling water directly hit on the cooler portion of the heat pipe and the supplied water temperature was changed. So if it could be ensured for a constant rate of supply and if the supplied water temperature remained constant, then a better performance may be achieved. Heat load increased as time increased. But after a certain time, it became steady with respect to time. The maximum heat load was obtained $2167.74 \mathrm{~W}$. From the figure 4 , it was seen that $\Delta \mathrm{T}_{1}$ increased as time increased. But there was quite a fluctuation in the figure. On the other hand from the figure 5, it was seen that though $\Delta \mathrm{T}_{2}$ increased with time, but there was less fluctuation in the figure than that of previous. Figure 6 shows the overall heat transfer. 


\section{CONCLUSION}

There was a numerous study of the straight heat pipe. But bent heat pipe is demanded in many practical sections where the source and sink are not in straight position. This study is to work with the heat transfer capacity of a bent heat pipe. In this work, copper pipe was used as a container. Copper pipe has high thermal conductivity. Since water is available everywhere, so it was used as working fluid. During the performance test, the cooling water was supplied by a long pipe which directly connected to the source. The cooling water directly hit the cooler section of the bent heat pipe and as the supplied water temperature was changed. So if it can be ensured of the constant rate of supply and if the supplied water temperature remains constant then a better performance may be achieved. In this work a wet cloth was used which surrounds the condenser region. From the experimental data, heat transfer was calculated. The maximum heat load was obtained $2167.74 \mathrm{~W}$. From literature review it is known that heat pipes have been studied mostly in the last couple of years. Actually, it is an efficient process of heat transfer. Using the sintered copper felt as wick material\& changing the bent angle in a heat pipe can be the upcoming work with more efficiency.

\section{REFERENCES}

Bienert, W. B., Brennan, P. J., "Transient Performance of Electrical Feedback Controlled Variable Conductance Heat Pipes", ASME Paper 71 Av 27, SAE/ASME/AIAA Life Support and Environmental Control Conference, San Francisco, California, July 12 $14,1971$.

Gaugler, R. S., "Heat Transfer Device", U. S. Patent 2, 350, 348.

Grover, G. M., Cotter, T. P. and Erikson, G. F., "Structures of Very High Thermal Conductivity", J. Appl. Phys., 35, 1990 (1964).

Grover, G.M., T.P. Cotter, and G.F Erickson (1964). "Structures of Very High Thermal Conductance ".Journal of Applied Physics35 (6): 1990-1991.

Hall, E.W. Saaski, "A flexible cryogenic heat pipe", AIAA 10 ${ }^{\text {th }}$ Thermophysics Conference, Denver, 1975.

Haskin, S. K.; Thomas, J Brown, "Development of a flexible loop heat pipe cold plate", SAE Aerospace Atlantic Conference, Dayton, 1995

Holman J. P., Heat Transfer, S I Metric Edition, McGraw-Hill.

Marcus, B. D., "Theory and Design of Variable Conductance Heat Pipes”, Reports no. 1and 2, TRW 13111 - 6027 - RO - 00 , Contract NAS 2 - 5503, April 1971.

Necati Ozisik M., Heat Transfer, A Basic Approach, McGraw-Hill.

Trefethen, L., "On the Surface Tension Pumping of Liquids or a Possible Role of the Candlewick in Space Exploration”, G. E. Tech. Info., Ser. No. 615 D114, Feb. 1962

Wyatt, Design and technology of heat pipes for cooling and heat exchange, Washington, D.C.: Hemisphere Pub. Corp., 1992 
